\title{
EXPLORING QUALITY OF SERVICE AND ITS CHALLENGES AT THE MOE PORT OF SPAIN OFFICE
}

Kit Fai Pun ${ }^{1}$ and Cherisse S. Lashley, ${ }^{2, *}$

Department of Mechanical and Manufacturing Engineering, The University of the West Indies, St Augustine, Trinidad and Tobago, West Indies

${ }^{1}$ Email: KitFai.Pun@sta.uwi.edu

2Email: scalashley@hotmail.com*

\begin{abstract}
In Trinidad and Tobago (T\&T), teachers often complain about the quality of service offered by the Ministry of Education (MOE). They generally describe it as poor because of the delays incurred during the processing of most requests. Poor service to customers (teachers) of an organisation implies a productivity deficiency. At MOE, requests made by teachers can turn into long waiting for the process to be completed or issues to be resolved. This has become a burden to teachers who are left to suffer the consequences as it appears little is being done to improve the system. To investigate the challenges associated with productivity issues and quality management (QM) practices at the MOE Port of Spain (POS) Office, a recent study was undertaken comprising of site visits and interviews with stakeholders (MOE staff and teachers) with the aid of online surveys. This paper presents the main findings of the survey, aiming to explore issues concerning QM processes, and identify the factors affecting the provision of quality services to teachers. The current quality culture and leadership styles of the MOE-POS Office would be addressed. The paper concludes by underlining the mechanisms of employee empowerment enabling a path to attain productivity/quality improvement, specifically for processing common requests or resolving issues at the MOE-POS Office.
\end{abstract}

Keywords: Productivity, quality, quality management, employee empowerment, education https://doi.org/10.47412/AYRK7960

\section{Introduction}

Quality can be a subjective term for which the definition is based on perception. Quality is derived from the clients' determination, experience, requirements, technical operations and it can take many forms. This topic is relevant because of the struggles and complaints from teachers concerning the service provided to them by their employment body, the Ministry of Education (MOE), at its main branch located in the capital of Port-of-Spain (POS). For decades, the Ministry has been functioning using an outdated system of processes. There have been processing times that remain untimely with communication confusions and disruptions between internal and external employees. Hence, requests could take months to be resolved which can be due to factors relating to the organisation's design and culture, leaving teachers as the external employees unsatisfied and annoyed by the organisations' overall service.

Nowadays, many quality initiatives in organisations fail because of disconnection to the top-level management strategy. The current issues that are prevalent in the Ministry suggest flaws in the 
organisation's approach to quality and productivity improvements. Decades have passed and teachers have been complaining about the slow process of request and the outdated system which relies heavily on a paper trail that would inevitably create lags in processing times at the Office. Although the organisation has recently made some improvements in processing requests, the most crucial processes still maintain a higher percentage of complaints from the teachers as one primary user of the MOE-POS Office's service. These complaints include delays in file updates, increments, salary increase, and permanent letters.

Productivity in an organisation is the ratio of the amount of output from a team per unit input. There are four types of productivity. Labour, capital, material and total productivity factor that are all important since they seek to answer a different set of questions in relation to quality. For this study, the focus is given to labour productivity since problems identified at the Ministry indicated that processing of the request is not carried out in a reasonable amount of time due to disruptions in labour. Moreover, contrast can be made to features of a quality service design, quality of performance that would satisfy the eight dimensions of quality (conformance, performance, serviceability, features, reliability, durability and aesthetics) [1] at the POS Office, to decipher the areas that require improvements.

The MOE is a governmental organisation. Its organisational structure models a matrix structure hence departments are cross-functional to each other. The MOE partners with the Service Commission (SC) who hires persons as 'public servants'. The Ministry's Office is separated into Civil and Education. Education is then separated into Primary and Secondary both of which have Human Resources (HR), Increment and Pay-sheet sections. This structure can be categorised into three levels of management, top, middle and low management who must commit to improving quality and productivity. Whether it is to implement firm management practices, use research and development effectively, and improve time management or; plan and coordinate quality and productivity efforts or; work with employees to adjust to change, to commit to quality and to continually improve all facets of their work.

\section{Literature Review}

\subsection{Productivity Management and Measures}

Productivity is the ratio of total output per unit input, that is one measure of how efficiently a system runs and uses its resources but it can be objective [2]. This definition focuses on words like efficiency, the ratio of outputs to inputs used and resources, but more questions should be asked to properly define the term. Questions such as 'what is the purpose?' and 'how can it be achieved most efficiently?' These answers were distorted over long periods by organisational culture, influenced by people and the organisational structure, which affects the productivity of an organisation, which also seems to be part of the dilemma at MOE. Efficiency depends on the comfort and fitness for work and the time to complete it. Since better results are achieved when employees enjoy the process and are efficient with their time, quality results are a side effect. Therefore, productivity can simply be defined as achieving quality results that contribute to the organisation's purpose [3]. This can only be cultivated into an organisation through a systematic process by maintaining a firm organisational structure with clear- cut goals and equilibrium, for instance, team building activities.

At the MOE-POS Office, the inputs would have been a request made by teachers and worker hours at the Office. Outputs would have been requests being completed. This step is the responsibility 
of Control - Process management to avoid saturation which is a problem at the Office. As productivity increases an organisation can find ways to turn resources into revenues. Simple changes to operations and processes can increase productivity [4]. However, some external processes affect productivity such as the production process (real and income distribution process) to be investigated and are dependent on the controllers of the organisation [3].

From an engineering aspect, movement towards increasing productivity of any organisation should be the focal point of the HR and involves planning to achieve a level of satisfaction [3]. Some factors affecting productivity on a national level are capital investments, economies of scale, workforce knowledge and skills resulting from training and experience, technological changes, work methods and procedures, systems, quality of results, quality of process, quality of management, legislative and regulatory environment, general levels of education, social environment and geographical factors. Of these factors, only the latter two is uncontrollable [4].

Since organisational culture and employee behaviour can be aligned to produce positive results. Training is a tool to develop knowledge and skills as a means of increasing an individual's performance (efficiency and effectiveness) apart from competitiveness and productivity. It has been found that employees would express discretionary effort if there is a proper Performance Management (PM) System supported by a compensation system. This brings us to job definition which clearly outlines the duties, responsibilities, working conditions and expected skills of the individual performing that job [5]. Productivity can only be measured indirectly by measuring other variables. This makes it difficult since inputs and outputs can be complex to define and quantify. A smart productivity measurement programme should produce a productivity index, and be achieved using performance rating at the MOE-POS Office.

\subsection{Quality Concepts and Dimensions}

Prominent professionals in the field have described quality as non-faulty systems or systems without errors that can provide the consumer with a product or service. Some keywords stand out when defining quality. Joseph M. Juran, describes quality as the fitness of use, whereas Philip Crosby discusses quality as conformance to requirements. Quality of design means the product or service has been designed successfully to fulfil the consumer needs, real or perceived. Quality of conformance refers to meeting the manufacturing specifications set by the consumer. Quality of performance means the product or service performs its intended function as identified by the consumer [1]. The critical dimensions of quality for strategic analysis of a quality framework are performance; conformance; features; reliability - probability of the product malfunctioning or failing within a specific period; durability - measure of product life which can be technical and economic; serviceability - speed, competence, and ease of repair; aesthetics refers the human senses and can be subjective as a matter of personal judgment and perceived quality - consumers may not have complete information on the product or service and hence indirect measures may be used based on comparing brands [6]. Moreover, analysing the processes from clients' viewpoint as measures to prevent functional barriers which is currently occurring between the service providers and teachers [1].

\subsection{Quality Culture, Principles and Empowerment}

Organisational culture refers to the taken-for-granted values, underlying assumptions, expectations, collective memories, and definitions present in an organisation. Quality culture in higher education is a kind of organisational culture that sustains the development of an effective 
and efficient quality management approach [6]. This approach allows the educational institution to realise its objectives and enhance the quality of its education and services [7]. Total Quality Management (TQM) was used as a reference to elaborate on quality culture since it strives towards continuous improvement in organisations [8]. Hence, quality is possible with coordination, communication, planning and that senior management has to take the lead to achieve quality [9]. Three core values can be contrasted from TQM principles [7]. These are:

1. Innovation, as a foundation for continuous improvement versus tradition,

2. Collective orientation, as a basis for working together to accomplish common goals versus individual specialisation, and

3. System control, as a basis for planned, systematic and coordinated quality control versus selfdetermination

If the quality of a service increases, there is less need for fixing mistakes, so productivity improves. Contrarily, it is claimed that if productivity increases so does the cost of production which ends with higher prices and reduced productivity. Moreover, productivity will increase, as well as quality and customer satisfaction achieved through continuous improvement [10].

Organisations can empower employees by providing them with a certain degree of autonomy and control on organisational tasks. It allows employees to have a better understanding of many of the basic processes and procedures that 'get the job done'. It can vary with the structure and culture of the organisation. Empowerment is built on the concepts of job enlargement and job enrichment in collaboration with job definition. [11].

\section{A 2-Phase Study Initiated at MOE-POS Office}

\subsection{Purpose and Design of Empirical Data Acquisition}

The purpose of this study was to investigate the reasons for delays when processing requests at the MOE POS Office. There are numerous pending requests at the Ministry involving teachers. The study addressed the struggles that teachers face in attempting to follow up with the hope of getting their matter advanced, but only to remain at the same stage of the process denoted as 'pending'. Reasons given for pending requests have always differed depending on the person on the other end of the phone call if the method of follow up was carried out that way. There is a productivity deficiency at the Office. There are three groups of client, internal employee, external employee and the public (students included). The employees are the focus in this study since they are the majority of whom receive ongoing service from the Office and also offer service to the public.

Statistics can be used to provide a sample report of teachers' reviews on the service received by the Ministry. The internal employees are a representation of the organisation and their performance output reflects the system by which they are managed. Therefore, two types of clients would be interviewed, namely the service provider and the receiver of service. For this reason, the study has two phases. This paper is concerned with the first phase that took the form of an online survey to collect information on teachers' experiences on productivity issues at the Office.

\subsection{Design and Refinement of Phase-1 Survey Instrument}

The online survey was undertaken to determine how many requests are being dealt with and how many have been completed, in addition to the processing time. It was designed to produce evidence 
that would be used to evaluate the claims made by teachers regarding service from the Ministry. The data collected would be classified as sample size data of the total number of teachers employed with the MOE either temporary or permanent. Table 1 describes the design of the survey.

Table 13. Design of the Phase-1 Teachers Survey

\begin{tabular}{|c|c|}
\hline Questions & Description \\
\hline $\begin{array}{l}\text { 1. Period of teaching } \\
\text { with MOE }\end{array}$ & This question indicates how long the teacher has been in the system. \\
\hline 2. Teacher Rank & $\begin{array}{l}\text { To determine the sections of the Ministry to be targeted for the second } \\
\text { survey. }\end{array}$ \\
\hline 3. Region of School & $\begin{array}{l}\text { This question indicates whether or not location affects the teacher's } \\
\text { ability to conduct follow-ups on their issues by visits. }\end{array}$ \\
\hline $\begin{array}{l}\text { 4. Routine of visits to } \\
\text { MOE POS Office }\end{array}$ & $\begin{array}{l}\text { This relates to question } 3 \text { as well as initial visits and creating a new } \\
\text { request. }\end{array}$ \\
\hline $\begin{array}{l}\text { 5. Completion of the } \\
\text { request upon visit }\end{array}$ & To identify overall service issues and productivity output \\
\hline $\begin{array}{l}\text { 6. Number of requests } \\
\text { still } \\
\text { processing/pending }\end{array}$ & Relates to question 5 and verify the level of productivity at the output. \\
\hline $\begin{array}{l}\text { 7. Types of request } \\
\text { which incur delays }\end{array}$ & To identify the specific requests that challenge productivity. \\
\hline $\begin{array}{l}\text { 8. Length of time to } \\
\text { process requests }\end{array}$ & To highlight the current waiting periods for requests to be completed. \\
\hline 9. Interests of teachers & To determine if follow-ups help accelerate resolution \\
\hline 10. Reasons for delays & To collect information on the possible causes of delay. \\
\hline 11. Overall service rating & $\begin{array}{l}\text { Although these questions are based on the respondents' knowledge of } \\
\text { quality and service in an organisation or productivity, it still provides a } \\
\text { general score for factual data from the receivers" end of the service. }\end{array}$ \\
\hline 12. Further suggestions & Proposed contributions. \\
\hline
\end{tabular}

\subsection{Determination of Population and Sample Size}

For Phase 1, the targeted group was the external employees of the MOE. From the stats collected, there are 13,259 primary and secondary school teachers employed in T\&T. Using a confidence interval (CI) of $95 \%$ or margin of error $5 \%$, and the target sample size of 373 was deduced.

\subsection{Execution and Analysis of Phase-1 Findings}

The survey was executed using social media platforms 'WhatsApp' and 'Facebook - A Teacher's Voice' since those were frequently used by teachers. The survey platform used generated basic analytics from the data collected. It is optional to perform advanced statistical analysis with the use of other software to obtain more detailed facts. From the analysis, implications were deduced and used to design the survey for Phase 2 of this study. 


\section{Findings and Analysis}

\subsection{Profile of Respondents}

From a sample of 337, respondents included persons who were employed by the MOE but don't work at the Office. They work as educators at schools governed solely or partially by the MOE but seek employee-related services from the POS Office. Those were active teachers, retirees and those on leave stationed at either north, south, east or west of T\&T. It was found that $67.94 \%$ are employed greater than 10 years with 4 respondents being retirees. Approximately $68 \%$ of the teachers visit the Ministry one to three times per year and $9.41 \%$ greater than six times per year as shown in Fig. 1. This implies that after the first visit teachers would most likely call to follow up. As depicted in Fig. 2, 47.94\% of the teachers are located in the south hence frequent visits greater than 3 would be a challenge to them hence phone calls to the Ministry to follow up on requests will increase. Although there is an MOE (district) Office in San Fernando (south), the south teachers prefer to visit the POS which is the main Office.

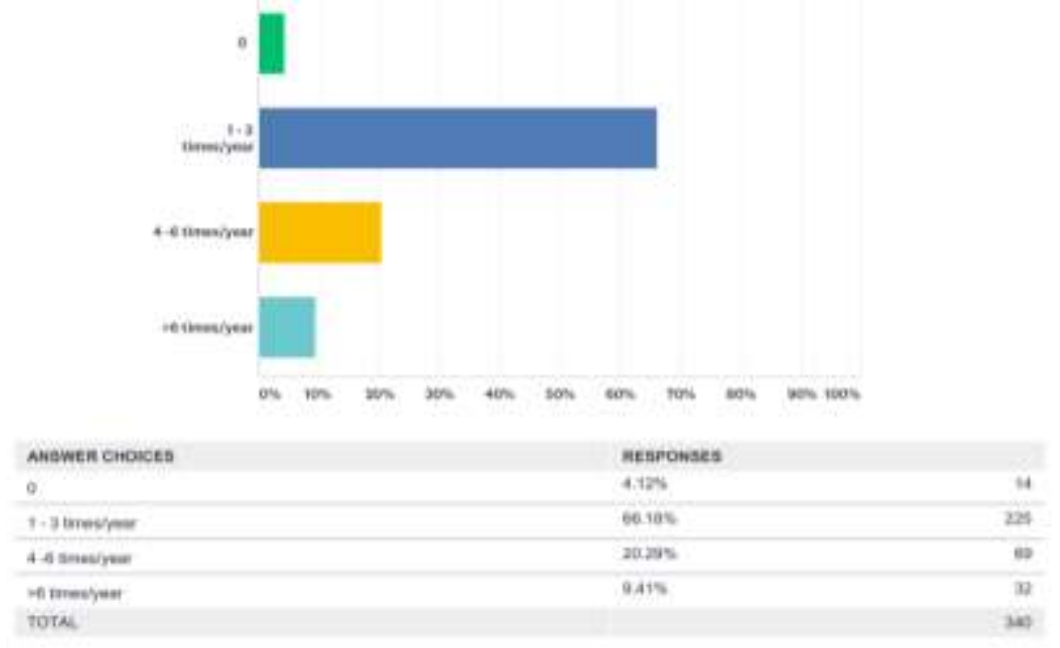

Figure 1. Teachers' Visits to the MOE-POS Office per year

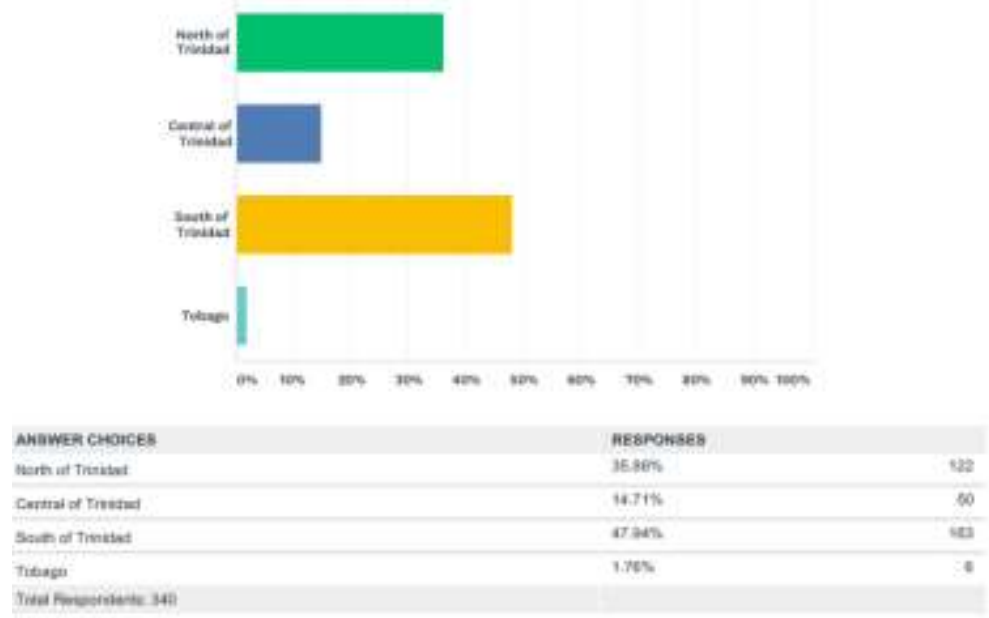

Figure 29. The Geographical Location of Respondents across Trinidad and Tobago 


\subsection{Completion of Service Requests (Productivity Outputs reported)}

Secondary school teachers took up $68 \%$ of the respondents with $78.24 \%$ leaving the Ministry Office without their service request completed (see Fig. 3). Evidence has shown the variety of requests in categories such as increments/arrears and job status upgrades, file record updates including appointment and confirmation letters, staff reports queries, medical and leave queries, misplaced files, pension and gratuity, and health insurance deduction queries. The greatest concern to teachers was their increments and arrears.

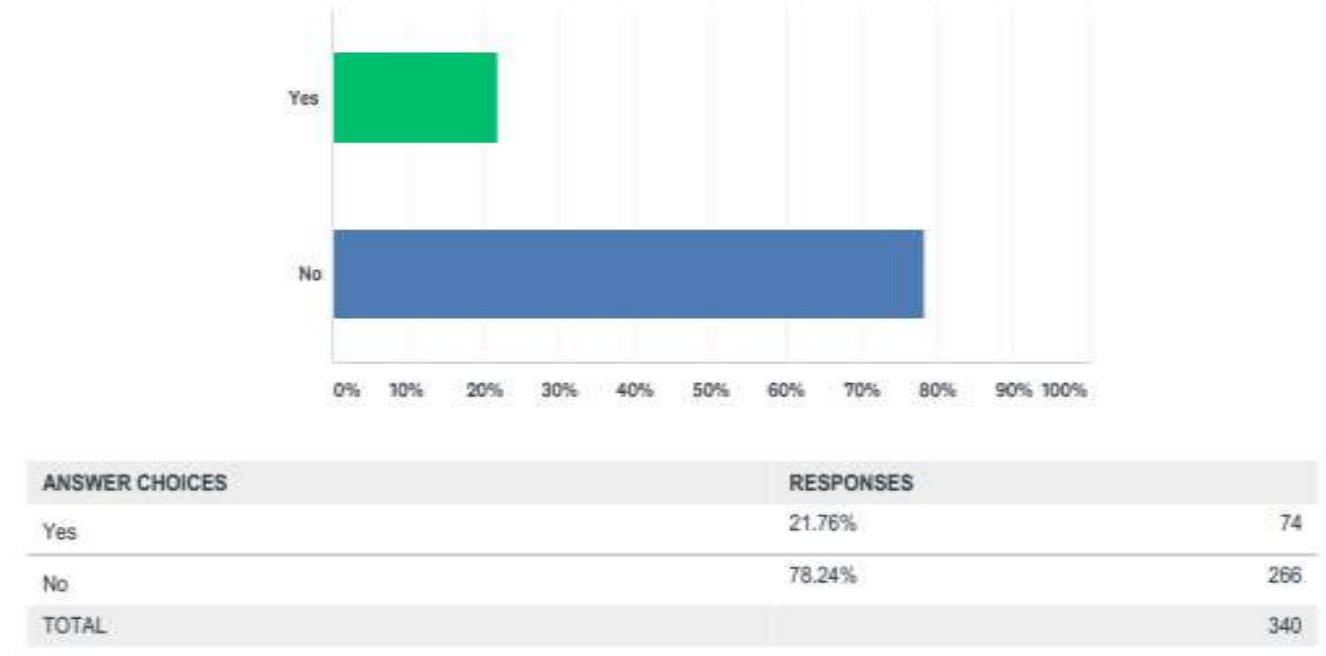

Figure 3. Completed Service by the MOE staff at POS

\subsection{Processing Times}

Of the teachers that visit the Ministry one to three times per year, $45.61 \%$ and $31.58 \%$ have a pending request for more than one year and greater than 5 years, respectively. This gives a total of $77.19 \%$ of unresolved requests greater than one year (see Fig. 4). It implies that productivity overall is low and is an indicator that HR practices are not being effectively implemented. Comparisons were made to the desk research and relations to questions identify factors of the problem. Several reasons for the delays as indicated by respondents, below:

- Calculation errors and mistakes by clerks

- Incorrect dates recorded on files

- The school has not been processed or late submission of staff reports by school administrators

- Letters have not reached the clerk

- Loss of reports/records

- Miscommunication between MOE and Teaching Service Commission

- Awaiting signatures from respective authorities

- Failure to provide follow up information to teachers

- Unpleasant attitude and ambiguous excuses

- Awaiting processing through the Teaching Service Commission

- Government has no funds

- The processing has not reached teachers' year of entry into the service or school.

- Different reasons for the delay of a specific request. 
- Ministry of Health and Ministry of Education correspondence delays

- Clerk irregularity and punctuality and failure to submitted documents

- Clerks are shuffled around between departments

- Staff uninformed of the procedures

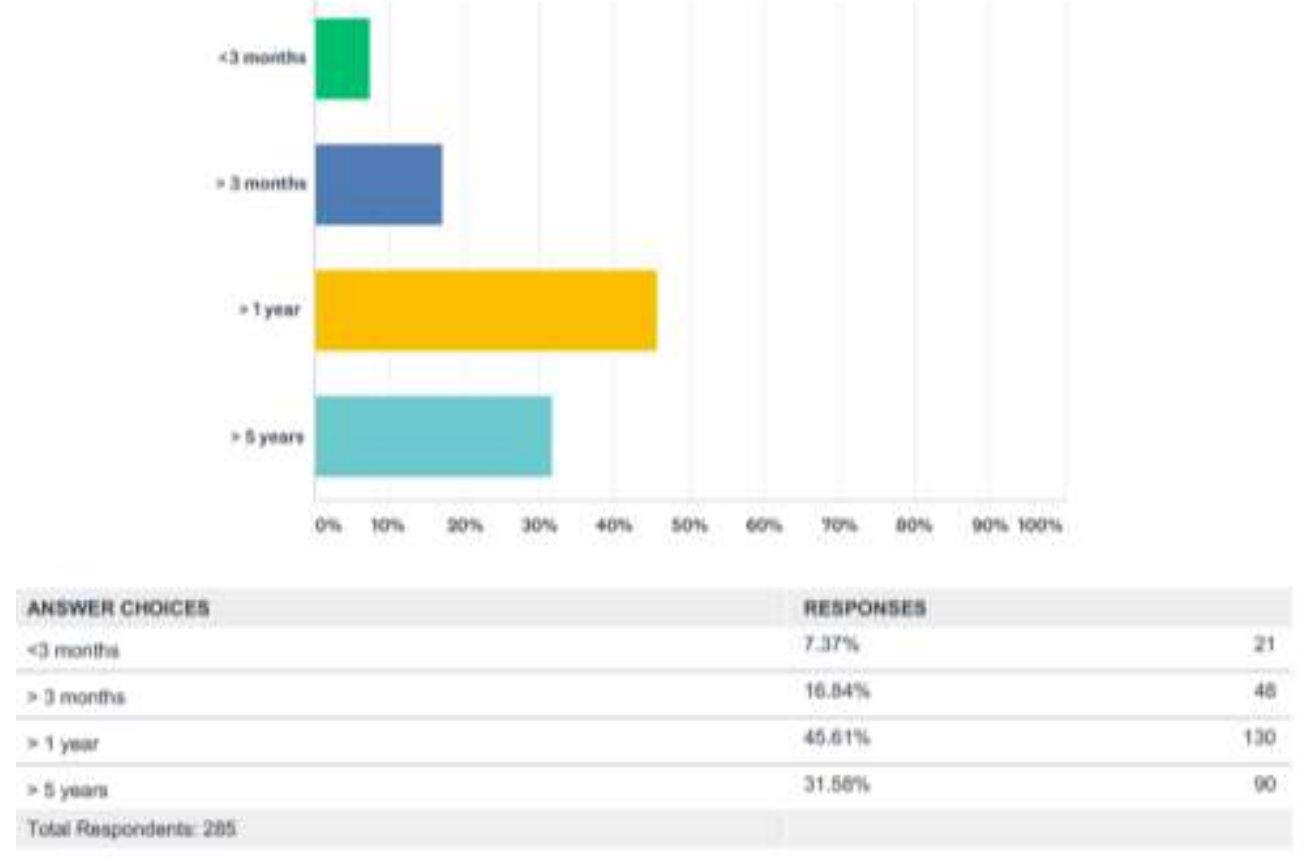

Figure 4. Time since the request has been pending

There were instances where errors were made and requests were not carried out correctly. Durability in the database is a property that ensures transactions are saved permanently. There were responses where teacher's files were lost or misplaced and therefore their request was not completed. The reliability of the current process wavered depending on the type of request. For instance, the request for a job letter is completed within 2-3 days but the request to update file or report can span greater than a year. Also, there is no standard time that a request is expected to be completed.

\subsection{Quality of Service rating by respondents}

Overall, $45.29 \%$ rated the service received by MOE staff as poor and $30.59 \%$ as unacceptable (see Fig. 5). This yielded a total of $75.88 \%$ rating service as below satisfactory level, with $22.65 \%$ rating as satisfactory and $1.47 \%$ rating their service as very good. 


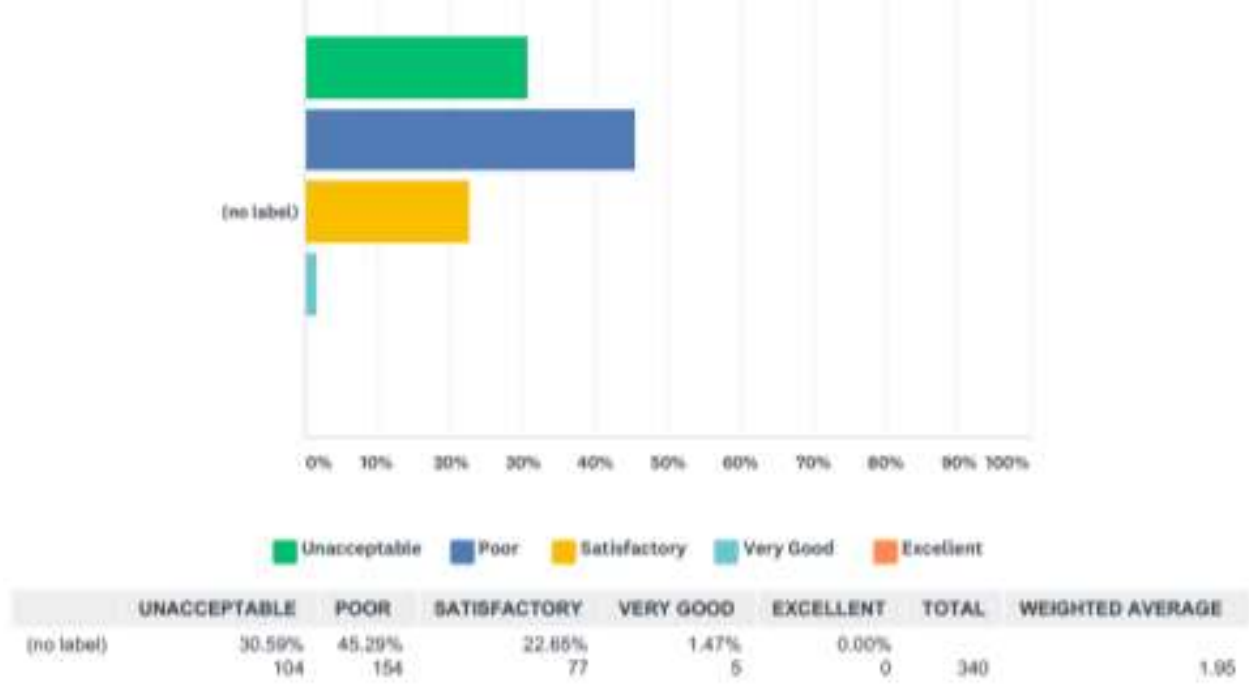

Figure 5. Rating of the MOE-POS Office Service by Respondents

\section{Discussions}

There are multiple routes for a department to fulfil a request. This gives way to several obstacles that can arise, as highlighted by teachers who proceeded to follow up on their requests. The reasons given for delays amounted to approximately seventeen which in summary suggests that the core values and paradigms of the organisation are weak, hence low productivity outputs and further, the dimensions of quality best practices are not established at the organisation.

It was found that the productivity has been at an all-time low due to the overall response rating. This rating was supported by reasons listed in Section 4.3 which highlighted the major issues affecting productivity measures and performance at the MOE POS Office. However, this only supports the subjective rating provided by the receivers of the service. Questions should then be asked about the people empowerment at the Office among the internal staff and the TQM principles that should be in practice that would increase this rating. For this reason, Phase 2 would be carried out.

Most of the factors affecting the output of productivity measures relied on training since the staff is uninformed about procedures (as discussed in Sections 4.2 and 4.3). Training would complement HR practices when implementing TQM through employee development of suitable skills (see Section 2.1). This indicates that not all employees are clear about their duties, responsibilities and expected skills. Employees must be clear about their job definition if not, it reflects the need to revise the PM System that may be supported by a compensation system. The presence of smart productivity would have yielded an acceptable productivity index through performance rating at the MOE POS Office (see Sections 2.2 and 4.4).

Also, the culture of the Office has impacted staff as professional values are taken for granted. For instance, punctuality, irregularity, and failure to submit documents have contributed to lags in 
processing times. In addition to 'clerk shuffle' between departments hence incorrect information for the reasons given for delays. Moreover, there is no follow-up procedure in place. Hence, a lot of the reasons given for delays are based on assumptions and collective memories. Furthermore, it can be suggested that management has not been meticulous in their strategies to normalise teams to obtain more cohesiveness and productivity. Team building activities are one way to empower individuals and enhances team effectiveness and can contribute to the success of the organisation.

\section{Conclusion}

The findings presented provides grounds for the HR practices to be revisited to improve the services offered to teachers in keeping with the best implementation of TQM. The empirical findings of Phase 1 would serve as an initiation to measure productivity from internal staff. It is recommended that the management system be revised to target productivity in keeping with the organisation's strategy and goals. It is suggested that an appraisal system be used to monitor employee performance within a standard period. In conjunction with the introduction of compensation packages for internal employees as a strategy to motivate staff to improve their performance. Also, an orientation programme where their job description is better defined or training workshops to better their skill to properly fulfil job requirements.

Although it is evident that other types of productivity are currently experiencing brawls, there are other areas of productivity such as labour and material that are dependent on controllers and can be targeted to boost overall productivity. The data also reflects that the inputs and outputs to productivity are not clearly defined to staff. The internal employees' performance relies on tradition since there is no clear indication of their job description. However, it can be introduced through orientation and training.

\section{Acknowledgements:}

The authors would like to thank for the Campus Research and Publication (CRP) Fund, for granting funds to this project (Ref. CRP.3.NOV17.5).

\section{References}

[1] Summers, D. C. S. Quality, NY: Pearson, 2018.

[2] LumenCandela, "Boundless Management," 1109 2019. [Online]. Available: https://courses.lumenlearning.com/boundless-management/chapter/managing-productivity/. [Accessed 2112020 ].

[3] Brand, Rob Van Den, "Structure and Process: Organisational Development - What is productivity?" Structure and Process, 28 May 2015. [Online]. Available: http://structureprocess.com/blog/what-isproductivity/. [Accessed 21 January 2020].

[4] Inman, R. A., "Productivity Concepts and Measures," Advameg, 2020. [Online]. Available: https://www.referenceforbusiness.com/management/Pr-Sa/Productivity-Concepts-andMeasures.html. [Accessed 22 January 2020].

[5] UK Essays, "Impact of HRM on Productivity," All Answers Limited, 13 July 2018. [Online]. Available: https://www.ukessays.com/essays/management/the-impact-of-hrm-practices-onemployees-productivity-management-essay.php\#citethis. [Accessed 22 January 2020]. 
[6] Reis, Elizabeth and Saraiva Margarida, "The Paradigm of the Total Quality Management in the Portuguese Higher Education," Proceedings of International Conference on Quality Assurance in Higher education: Mission (Im)possible? Pretoria - South Africa, 2006.

[7] Berings, D., "Reflection on quality culture as a substantial element of quality management in higher education," in Creativity and Diversity: Challenges for quality assurance beyond 2010, Copenhagen, 2009.

[8] Wilkinson J., "Total quality management definition," 24 July 2013. [Online]. Available: https://strategiccfo.com/total-quality-management/.

[9] Gotham Culture, "Strategic Planning," 2019. [Online]. Available: https://gothamculture.com/services/strategy-consulting/strategic-planning/. [Accessed 9 August 2019].

[10] Kontoghiorghes C., "Examining the association between quality and productivity performance in a service organization." Quality Management Journal, vol. 10, no. 1, January 2003.

[11] American Society of Quality, "Employee Empowerment," 2018. [Online]. Available: https://asq.org/quality-resources/employee-empowerment. [Accessed 19 August 2019]. 